\title{
A random survey of the prevalence of falsified and substandard antibiotics in the Lao PDR
}

\author{
Patricia Tabernero $\mathbb{D}^{1-3, *}$, Isabel Swamidoss ${ }^{4}$, Mayfong Mayxay ${ }^{1,5,6}$, Maniphone Khanthavong ${ }^{7}$, \\ Chindaphone Phonlavong ${ }^{8}$, Chanthala Vilayhong ${ }^{1}$, Sengchanh Yeuchaixiong ${ }^{1}$, Chanvilay Sichanh ${ }^{1,2}$, \\ Sivong Sengaloundeth ${ }^{9}$, Michael D. Green ${ }^{4}$ and Paul N. Newton ${ }^{1,2,5,10,11}$
}

\begin{abstract}
${ }^{1}$ Lao-Oxford-Mahosot Hospital-Wellcome Trust Research Unit (LOMWRU), Microbiology Laboratory, Mahosot Hospital, Vientiane, Lao PDR; ${ }^{2}$ WorldWide Antimalarial Resistance Network (WWARN), Oxford, UK; ${ }^{3}$ Public Health Unit, Faculty of Medicine, University of Alcalá, Alcalá de Henares, Spain; ${ }^{4}$ U.S. Centers for Disease Control and Prevention (CDC), Division of Parasitic Diseases and Malaria, Atlanta, GA, USA; ${ }^{5}$ Centre for Tropical Medicine \& Global Health, Nuffield Research Building, Churchill Hospital, University of Oxford, Oxford, UK;

${ }^{6}$ Institute of Research and Education Development, University of Health Sciences, Vientiane, Lao PDR; ${ }^{7}$ Centre of Malariology, Parasitology and Entomology (CMPE), Vientiane, Lao PDR; ${ }^{8}$ Bureau of Food and Drug Inspection (BFDI), Ministry of Health, Government of the Lao PDR, Vientiane, Lao PDR; ${ }^{9}$ Food and Drug Department (FDD), Ministry of Health, Government of the Lao PDR, Vientiane, Lao PDR; ${ }^{10}$ Infectious Diseases Data Observatory, Nuffield Research Building, Churchill Hospital, University of Oxford, Oxford, UK; ${ }^{11}$ Faculty of Infectious and Tropical Diseases, London School of Hygiene and Tropical Medicine (LSHTM), London, UK
\end{abstract}

*Corresponding author. Tel: +34 918225393; E-mail: patriciataberneroestevez@gmail.com (1) orcid.org/0000-0002-1015-6207

Received 19 December 2018; returned 14 January 2019; revised 18 March 2019; accepted 24 March 2019

Objectives: In 2012, a stratified random survey, using mystery shoppers, was conducted to investigate the availability and quality of antibiotics sold to patients in the private sector in five southern provinces of the Lao People's Democratic Republic (Laos).

Methods: A total of 147 outlets were sampled in 10 districts. The active pharmaceutical ingredient (API) content measurements for 909 samples, including nine APIs (amoxicillin, ampicillin, ceftriaxone, ciprofloxacin, doxycycline, ofloxacin, sulfamethoxazole, tetracycline and trimethoprim), were determined using HPLC.

Results: All the analysed samples contained the stated API and we found no evidence for falsification. All except one sample had all the units tested with \%API values between $75 \%$ and $125 \%$ of the content stated on the label. However, we identified the presence of substandard antibiotics: 19.6\% (201/1025) of samples had their units outside the $90 \%-110 \%$ content of the label claim and $60.2 \%$ (617/1025) of the samples had units outside of the International Pharmacopoeia uniformity of content limit range. Amoxicillin had a high number of samples [67.1\% (151)] with units above the limit range, followed by ciprofloxacin [58.8\% (10)] and ofloxacin [57.4\% (39)]. Ceftriaxone, trimethoprim and sulfamethoxazole had the highest number of samples with low API content: $57.1 \%(4), 51.6 \%(64)$ and $34.7 \%$ (43), respectively. Significant differences in \%API were found between stated countries of manufacture and stated manufacturers.

Conclusions: With the global threat of antimicrobial resistance to patient outcomes, greater understanding of the role of poor-quality antibiotics is needed. Substandard antibiotics will have reduced therapeutic efficacy, impacting public health and control of bacterial infections.

\section{Introduction}

Access to good-quality medicines is a critical factor for the effective management and control of diseases globally and universal health coverage. ${ }^{1}$ The increased accessibility and inappropriate use of antimicrobials have led to enhanced selective pressure and development of resistant pathogens. Antimicrobial resistance threatens the effective prevention and treatment of infections in both the developed and developing world, and is growing at an alarming pace. . $^{-5}$

Factors contributing to the development of antibiotic resistance include inappropriate use of antibiotics due to poor prescribing and patient adherence. Furthermore, poor storage conditions may result in physicochemical changes causing degradation or altered dissolution of active ingredients. In addition, subtherapeutic amounts of active pharmaceutical ingredients (APIs) and poor API

(o The Author(s) 2019. Published by Oxford University Press on behalf of the British Society for Antimicrobial Chemotherapy.

This is an Open Access article distributed under the terms of the Creative Commons Attribution License (http://creativecommons.org/licenses/ by/4.0/), which permits unrestricted reuse, distribution, and reproduction in any medium, provided the original work is properly cited. 
dissolution of antimicrobials engender resistance for some pathogens. ${ }^{6}$ Resistance is most likely to develop when pathogens are exposed to low API concentrations, high enough to kill susceptible organisms, but not resistant ones. ${ }^{6-9}$

Although there is a logical relationship, quantification of the contribution made by poor-quality medicines to antimicrobial resistance remains unknown due to the lack of information and understanding. Few objective data on the prevalence of poorquality antibiotics exist. Some research groups and international NGOs have tried, over the last two decades, to estimate their prevalence and highlight the problem in developing countries. ${ }^{10-20}$ Examples include a study conducted in the late 1990s in Nigeria and Thailand, showing that $36 \%$ of the samples collected in Nigeria and $40 \%$ of samples collected in Thailand contained quantities of APIs outside pharmacopoeial limits. ${ }^{21}$ The death of a patient from bacterial meningitis was associated with substandard ceftriaxone in Uganda in 2013. ${ }^{13}$ The strong demand for antibiotics runs the risk of creating a market for falsification, ${ }^{12}$ and they are widely falsified. ${ }^{10}$ There is increasing awareness that poor-quality medicines are important impediments to public health. ${ }^{14,22-25}$ Poor-quality medicines include those falsified and those which are substandard (from errors in manufacture) or degraded (in the supply chain). The latter two categories are included together in the 2017 WHO definitions. ${ }^{26}$

The problem is aggravated by the lack of testing facilities in low- and middle-income countries (LMICS) and poor data sharing. ${ }^{27}$ Misdiagnosis and antimicrobial resistance are usually considered the main reasons for treatment failure, without consideration of the risk of poor-quality medicines (and, consequently, investigations on the latter are not prompted).

Reports in the Greater Mekong subregion have demonstrated high frequencies of falsified and substandard antimalarials, ${ }^{24,28-33}$ but there are few data on antibiotic quality in the public domain. Surveys conducted in the late 1990s investigated the availability of poor-quality antibiotics including ampicillin and tetracycline in the Lao PDR (LaOs). ${ }^{31,34}$ Operation Storm I and II, conducted in 200809 , showed that $31 \%$ of seized antibiotics analysed were of poor quality. ${ }^{35}$

However, very few data were acquired using random sampling that would allow objective estimates of the proportion of a nation's or a province/region's antibiotic supply that is substandard or falsified, and there are none from Laos. ${ }^{36-38}$ Data on antibiotic resistance in Laos are scarce, but ESBL Enterobacteriaceae are becoming more frequent. ${ }^{39-41}$

In 2012, we conducted a survey to investigate the availability and quality of selected antimalarials and antibiotics sold to patients in the private sector in five southern Lao provinces. The study methodology and results for antimalarials have been described, ${ }^{37}$ and here we report the results on the quality of the antibiotics collected.

\section{Methods}

\section{Setting}

Laos has a population of $\sim 6.8$ million people, with the majority $(60.3 \%)$ living in rural areas. ${ }^{42-45}$ One urban and one rural district (i.e. stratified by urbanization) were selected using simple random selection, by random number tables, from each of the five southern provinces, and all known outlets in these districts were sampled. ${ }^{37}$ The districts selected were Adsaphangthong and Sepon in Savannakhet Province, Salavan and
Toumlane in Salavan Province, Sekong and Thateng in Sekong Province, Sammakkyxay and Sanamxay in Attapeu Province, and Pakse and Sanasoumboun in Champasak Province (Figure S1, available as Supplementary data at JAC Online).

\section{Study design}

A cross-sectional random sampling of private sector medicine outlets was conducted in the five southern Lao provinces starting in September 2012, lasting 4 weeks. One male and one female research assistant from Vientiane purchased, as mystery shoppers, the anti-infective medicines from all private retail outlets identified in the selected districts. Prior to the survey, a 1 week training was conducted in Vientiane. This included pretesting of the data collection tools and the debriefing process.

Outlets were visited twice, first by a mystery shopper who stated that they were a friend of a sick malaria patient working in construction in southern Lao (for the antimalarial medicine quality survey ${ }^{37}$ ) and, secondly, by another mystery shopper with a handwritten list of essential antibiotics and anti-TB medicines (Text S1). Visits were conducted one day apart. Twenty tablets/capsules of each preparation of amoxicillin, ampicillin, ciprofloxacin, co-trimoxazole, ofloxacin, tetracycline and doxycycline were requested. Rifampicin, isoniazid, ethambutol and pyrazinamide were also requested.

If no staff were present at the first visit, two further attempts were made to visit outlets. Hand-held GPS (Global Positioning System) units were used to map outlet locations (within $\pm 10 \mathrm{~m}$ ). Debriefing of the mystery shopper was conducted after each outlet interaction on the same day using a semi-structured questionnaire (Form S1).

\section{Inclusion criteria}

All private pharmacies, private clinics or medicine sellers in the study districts, whether registered or unregistered, were eligible for inclusion in the survey. Private pharmacies are classified depending on the qualifications of the licensee: ${ }^{31,46}$ class I pharmacies are run by a qualified pharmacist with a university degree; class II pharmacies are run by an assistant pharmacist; and class III pharmacies are run by any medical professional, usually an auxiliary nurse or a low-level pharmacist. Registered Private Clinics are run after Government working hours by medical doctors. These are licensed to sell antibiotics.

Poor-quality medicines were defined as falsified or substandard based on WHO definitions without consideration of intellectual property issues. ${ }^{26}$ A sample was defined as a group of apparently physically identical dosage units (e.g. tablets or vials), from one brand and one batch obtained at the same time from the same outlet. Samples were kept in a foam box and sent to Vientiane within 3-4 days of collection, for storage in a refrigerator at $+4^{\circ} \mathrm{C}$ before shipment for analysis.

All data were double-entered in a pre-established Epi-Data database. Data were analysed using STATA (v11.2, Stata Corp, College Station, TX, USA), RStudio Version 1.0.136 (RStudio Team, 2016) and Microsoft Excel.

\section{Laboratory analysis of the samples}

Anti-infective samples were sent for chemical analysis to the CDC in Atlanta, USA. Analysis was completed 24 months after sample collection. The API content measurements for each sample were determined using HPLC. ${ }^{47}$ Between one and three dosage units were tested for each sample (when available) and the mean of the percentage API, with reference to the stated dose on the packaging, was calculated.

Samples were primarily classified as meeting the quality requirements if the amount of API in each of the units, as determined from their content uniformity, ${ }^{48-52}$ lay within the range of the International Pharmacopoeia and/or British Pharmacopoeia percentage of the label claim; see Table S1. Typically, pharmacopoeial content and uniformity methods require at least 
20 units for content analysis and at least 10 units for stage one dose uniformity analysis. ${ }^{53}$ Using a lower number of units may thus under- or overestimate the conclusion. Since the numbers of units collected were limited and given the heterogeneity of within-specification threshold ranges between the different pharmacopeias (Table S1), we also categorized each unit using the $85.0 \%-115.0 \%$ and the $75.0 \%-125.0 \%$ API threshold ranges.

Packaging analysis was conducted in comparison with the genuine medicine when these were available, blinded to chemistry, by visual inspection and using the U.S. FDA CD-3. ${ }^{54}$ The CD-3 is a handheld device that uses different wavelengths of light to compare an authentic medical product and packaging with a potentially falsified medicine and its packaging.

As $31 \%$ of samples were analysed after their expiry date, to better understand differences in \%API in relation to the medicine's expiry date, a regression analysis of \%API versus days until expiry (defined as expiry date-date of analysis) was conducted using a two-parameter decay equation [ $y=a \times \exp (-b x), y=\% A P I, x=$ days till expiry]. Sigmaplot 12.0 was used to calculate the non-parametric Spearman correlation value and the associated $P$ value. The \%API was normalized using the rate constant ' $b$ ' from the equation to compensate for any possible API degradation due to analysing samples past their expiry date.

This report has been written following the Medicine Quality Assessment Reporting Guidelines (MEDQUARG), and the results have been reported to the Lao FDD and WHO RapidAlert. ${ }^{55-57}$

\section{Ethics}

Ethical clearance was granted by the Lao PDR National Ethics Committee for Health Research (NECHR); approval reference number 054.

\section{Results}

\section{Survey description}

A total of 147 outlets were sampled in the 10 districts, 45 in the rural and 102 in the urban districts. Three outlets were closed and therefore 144 (98\%) were included in the analysis (Figure S1 and Figure S2).

Registered outlets accounted for $97.2 \%$ (140) of those included. Pharmacy classes I and II accounted for 30.9\% (43) and 30.9\% (43) of outlets, respectively, and $33.8 \%$ (47) were pharmacy class III. ${ }^{31,46}$ Only $4.1 \%$ (6) outlets were registered clinics, and one of the outlets sampled was a shop of a registered pharmaceutical manufacturer, Pharmaceutical Factory No. 2. Only four unregistered outlets (2.7\%) were found, and they were general shops that were also selling medicines.

Antibiotics were bought from $96.5 \%$ (139) of the included outlets. Mystery shoppers were unable to buy antibiotics in five outlets. The provider was absent in one outlet and four did not have antibiotics in stock.

No provider requested to see a medical prescription.

\section{Medicines offered to mystery shoppers}

A total of 1173 medicine samples were collected and, of those, 158 were antimalarials and 1015 were medicines for the treatment of fever and TB and not for malaria.

Out of the 1015 medicine samples sold for the treatment of fever (as claimed by outlet staff), 95.5\% (969) were labelled as antibiotics and $4.5 \%$ (46) were labelled as other types of medicines such as paracetamol, antihistamines and vitamins (Figure S3 and Table S2).
Of the antibiotics collected, $15.6 \%$ (151/969) of samples were sold as loose units of only one type of medicine in plastic bags with no label or patient information, stated manufacturer or expiry date, and, of those, $84.7 \%$ (128) had no trade name and $47.7 \%$ (72) had no dosage information. Of these 128 plastic bags, $88.3 \%$ (113) capsule samples were sold as containing tetracycline, 3.1\% (4) capsule samples as containing doxycycline, $2.3 \%$ (2 tablets and 1 capsule) as containing ampicillin, $1.6 \%$ (2) samples as ciprofloxacin tablets, $1.6 \%$ (2) as chloramphenicol capsules, $1.6 \%$ (2) as isoniazid tablets, $0.7 \%$ (1) as amoxicillin capsules and $0.7 \%$ (1) as ofloxacin tablets. Only 9.6\% (93/969) of the samples were sold with secondary packaging (i.e. boxes or containers) and $11.8 \%$ (115/969) of samples gave storage instructions on the package or in a leaflet.

Eight $(0.8 \%)$ samples had expired at the time of sample collection and a further 308 (31.8\%) by the time chemical analysis was conducted.

Ampicillin $(26.5 \%, 257)$ and amoxicillin $(23.4 \%, 227)$ were the medicines most frequently sold, accounting for half of the antibiotics collected. Other antibiotics collected include sulfamethoxazole/ trimethoprim $(12.9 \%, 125)$, tetracycline $(12.1 \%, 117)$, doxycycline $(9.0 \%, 87)$, ofloxacin $(7.0 \%, 68)$, ciprofloxacin $(1.9 \%, 18)$, cefalexin $(1.2 \%, 12)$, norfloxacin $(1.2 \%, 12)$, intravenous ceftriaxone $(1.0 \%$, 10 ) and four (0.4\%) samples of chloramphenicol (Table S2).

Anti-TB monotherapy was collected in 13 (9.0\%) outlets, consisting of $10(1.0 \%)$ samples of rifampicin, $6(0.6 \%)$ samples of isoniazid and $4(0.4 \%)$ samples of ethambutol. These were not analysed chemically.

There were 145 branded products from 41 stated manufacturers of which $25.5 \%$ (37) were registered with the Lao Food and Drug Department, using the list of 2012 . Of the $86.4 \%$ (886) antibiotic samples that specified a manufacturer, $25.3 \%$ (259) were labelled as made by 'Codupha-Lao Pharmaceutical Factory, Vientiane, Lao P.D.R.', 19.2\% (197) were labelled as made by 'CBF Pharmaceutical Factory, Pakse-Champasack, Lao P.D.R.' and 12.8\% (132) were labelled as made by 'KPN Pharma Co., Ltd, Vientiane, Lao P.D.R.'

Samples were labelled as manufactured in seven countries, with most of them $(58.7 \%, 602)$ labelled as made in Laos. Other countries stated as the origin were India (11.5\%, 118), China (7\%, $72)$, Thailand $(4 \%, 41)$, Vietnam $(3.7 \%, 38)$, South Korea $(0.2 \%, 2)$ and Bangladesh $(0.1 \%, 1)$. For $14.7 \%(151)$ of the samples, the country of manufacture was not stated.

CD-3 analysis could be conducted on 345 samples as genuine comparators were not available for $62.0 \%$ (564) of the medicines sampled. Of those, $56.2 \%$ (194) of the samples failed packaging analysis; $61.2 \%$ (71) of the tetracycline samples collected had the same tablet design and were consistent with each other under the CD-3 light, but were not consistent when analysed against the only genuine comparator available. No correlation between failing visual inspection and failing chemical analysis was found $(P=0.056)$.

A total of $25.11 \%$ (57) of the amoxicillin, $16.9 \%$ (7) of the sulfamethoxazole/trimethoprim, $15.5 \%$ (40) of the ampicillin, $13.7 \%$ (12) of the doxycycline, $11.1 \%$ (2) of the ciprofloxacin and $7.3 \%$ (5) of the ofloxacin samples failed packaging analysis.

\section{Chemical quality of the antibiotics}

Of the 969 antibiotics collected, 909 samples and nine APIs (amoxicillin, ampicillin, ceftriaxone, ciprofloxacin, doxycycline, 


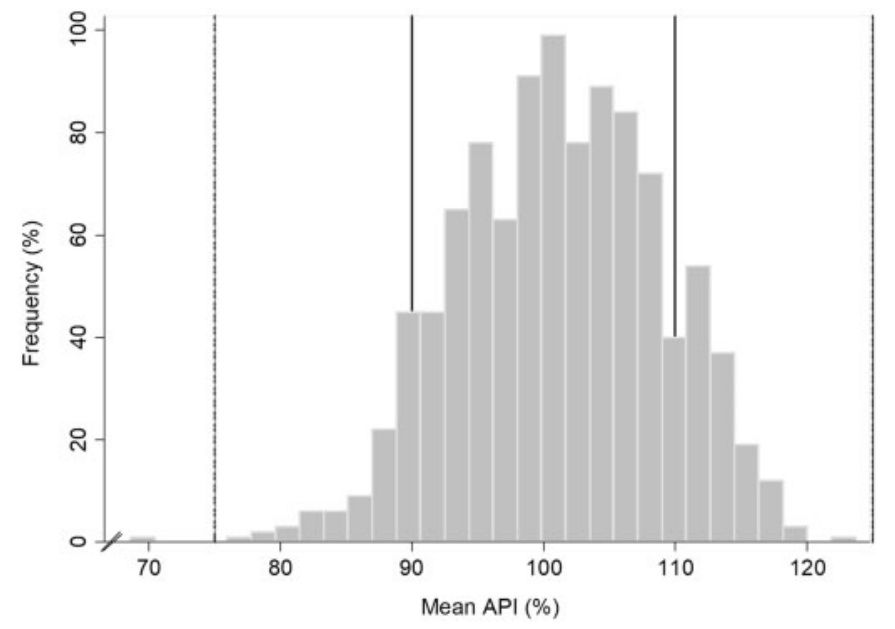

Figure 1. Frequency of antibiotic mean \%API found in the samples $(n=1025)$. The two outer lines represent $75 \%$ and $125 \%$ cut-offs and the two inner lines represent $90 \%$ and $110 \%$ pharmacopoeial \%API limits.

ofloxacin, sulfamethoxazole, tetracycline and trimethoprim) were analysed; 60 samples stated as containing other APIs were not analysed (Figure S3 and Table S3).

Out of the 1034 APIs analysed (909 samples plus 125 trimethoprim from the co-formulated sulfamethoxazole/trimethoprim), 9 samples were lost during analysis. All the 1025 API samples analysed labelled as antibiotics contained the stated API.

All except one sample had all the units tested with \%API between $75 \%$ and $125 \%$ of the content stated on the label. Most of the samples $(96.1 \%, 985)$ had their units' mean \%API between $85 \%$ and $115 \%$, and $80.4 \%$ (824) had their units' mean between $90 \%$ and $110 \%$ (Figures 1 and 2 ).

The sample with \%API outside the range $75 \%-125 \%$ was a sulfamethoxazole/trimethoprim co-formulated syrup (stated as manufactured by PDC, Pharmaceutical Factory No. 3, Laos) in which one of the two sulfamethoxazole units analysed had a \%API below the $75 \%$ cut off (API values: $60 \%$ and $77 \%$ per sulfamethoxazole unit tested; $82 \%$ and $102 \%$ per trimethoprim unit tested).

In total, 39.8\% (408) of the samples had all their units within the API-specific limit range of the International Pharmacopoeia uniformity of content assay (Table 1 and Table S1).

Ampicillin sodium in injection form was the API with the highest proportion of samples with all the units within the International Pharmacopeia specifications $(80.8 \%, 84)$, followed by sulfamethoxazole with $61.3 \%$ (76) samples, doxycycline with $56.5 \%$ (48) samples and amoxicillin trihydrate with $50.0 \%$ (1) samples (Table 1).

Samples of ampicillin trihydrate (1) and doxycycline hyclate (2) had all their units outside the International Pharmacopeia specifications.

Of the 124 co-formulated sulfamethoxazole/trimethoprim samples analysed, $42.7 \%$ (53) had both APIs within the limit range.

Of the $60.2 \%$ (617) samples that were outside the \%API specifications of the International Pharmacopeia limit range, 62.7\% (387) samples had units with higher amounts of API, 33.2\% (205) samples had lower amounts of API and a significant minority 4.0\% (25) of samples contained units in samples both above and below the limits (Table 2).

Inter-tablet variability was also measured for up to three tablets per dosage unit from the same sample. Ciprofloxacin had the highest variability between its units, with mean relative standard deviation (RSD) of 5.2 (Figure 3, Table S4 and Table S5).

Of the samples with units outside the pharmacopoeia limit ranges, a significant difference was found between the stated country of manufacture and the \%API of the sample (KruskalWallis $P=0.0001$ ); $60.3 \%$ (372) of the failed samples were labelled as being made in Laos, $15.4 \%$ (95) with missing manufacturer, $11.8 \%$ (73) from India, 5.5\% (34) from China, 4.1\% (25) from Thailand, $2.6 \%$ (16) from Vietnam, 0.1\% (2) from South Korea and $0.1 \%$ (1) from Bangladesh. The API failure frequency was significantly associated with the stated manufacturer (Kruskal-Wallis $P=0.0001$ ).

Half of the samples stated as manufactured in Vietnam, China and South Korea were within the limit range $(57.9 \%, 52.8 \%$ and $50 \%$, respectively) and only $39.2 \%, 38.2 \%$ and $38.1 \%$ of the samples labelled as manufactured in Thailand, Laos and India had all units of acceptable quality.

\section{Stability}

Stability plots revealed trends in API degradation with days until expiry. Characterization of the variation of the \%API in relation to the medicine's remaining time to expiry was determined by regression analysis (Figure 4).

In this figure, dashed lines represent time trends associated with degradation in \%API content. The \%API of medicines with significant slopes $(P<0.05)$ was normalized by adjusting the slope to zero to compensate for these changes and is represented by the solid line.

Stability plots demonstrate changes in \%API in time before and after the expiry date. Trimethoprim, ciprofloxacin and amoxicillin ampoules showed a weak to moderate correlation between \%API reduction and expiry date $(P<0.05$; Figure 4$)$. For ciprofloxacin tablets and amoxicillin ampoules, \%API significantly declined with increased sample age, but the reverse was found for trimethoprim.

\section{Discussion}

Despite their key importance for treating infections, little is known in the public domain about the quality of antimicrobials in the Greater Mekong subregion, notwithstanding the significant anecdotal evidence that poor-quality antibiotics are present in south east Asia and elsewhere. ${ }^{10,11,23,28,29,34,58,59}$

All samples contained the stated API and most of the samples contained the correct mean amount of API, although there was a significant variation in the quantity of active ingredient within the samples. Results obtained from these antibiotics are consistent with the findings of the quality of antimalarials collected in the same survey. ${ }^{37}$

There was only one sample (sulfamethoxazole/trimethoprim co-formulated syrup) for which the dosage units contained \%API $<75 \%$, but there was no genuine comparator available to 


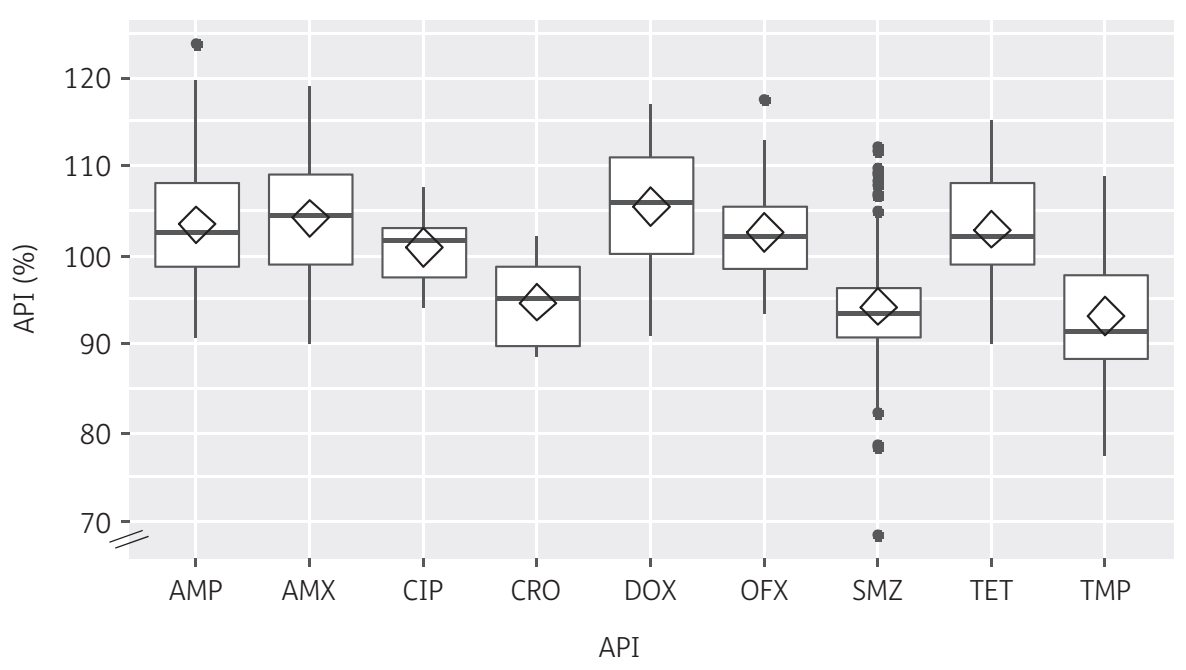

Figure 2. Box plot showing the mean API (\%) by medicine. It includes both intravenous and oral forms. Diamonds represent mean values and horizontal lines represent median values. Box borders represent the lower and upper quartiles (25th and 75th percentiles, respectively). AMP, ampicillin ( $n=256)$; AMX, amoxicillin ( $n=225)$; CRO, ceftriaxone ( $n=7)$; CIP, ciprofloxacin ( $n=17)$; DOX, doxycycline $(n=87)$; OFX, ofloxacin ( $n=68)$; SMZ, sulfamethoxazole $(n=124)$; TET, tetracycline $(n=117)$; TMP, trimethoprim $(n=124)$.

Table 1. Quality of the samples surveyed classified by the International Pharmacopoeia uniformity of content limit range

\begin{tabular}{|c|c|c|c|c|c|c|c|}
\hline \multirow{2}{*}{$\begin{array}{l}\text { Medicines surveyed, } \\
\text { labelled API }\end{array}$} & \multirow{2}{*}{$\begin{array}{c}\text { Analysed } \\
\text { samples, no. }\end{array}$} & \multirow{2}{*}{$\begin{array}{c}\text { Lost } \\
\text { samples, no. }\end{array}$} & \multirow{2}{*}{$\begin{array}{l}\text { International } \\
\text { Pharmacopoeia } \\
\text { specifications of limit } \\
\text { range, \% content }\end{array}$} & \multicolumn{2}{|c|}{$\begin{array}{l}\text { Samples with all } \\
\text { units within limit range }\end{array}$} & \multicolumn{2}{|c|}{$\begin{array}{l}\text { Samples with units } \\
\text { outside limit range }\end{array}$} \\
\hline & & & & no. & $\%$ & no. & $\%$ \\
\hline Amoxicillin anhydrous & 15 & & $95-102$ & 3 & 20.0 & 12 & 80.0 \\
\hline Amoxicillin & 152 & 1 & $95-102$ & 21 & 13.8 & 131 & 86.2 \\
\hline Amoxicillin sodium & 56 & & $95-102$ & 5 & 8.9 & 51 & 91.1 \\
\hline Amoxicillin trihydrate & 2 & 1 & $95-102$ & 1 & 50.0 & 1 & 50.0 \\
\hline Ampicillin trihydrate & 1 & & $95-102$ & 0 & 0.0 & 1 & 100.0 \\
\hline Ampicillin & 151 & & $95-102$ & 53 & 35.1 & 98 & 64.9 \\
\hline Ampicillin sodium intravenous & 104 & 1 & $90-110$ & 84 & 80.8 & 20 & 19.2 \\
\hline Ceftriaxone & 7 & 3 & $96-102$ & 3 & 42.9 & 4 & 57.1 \\
\hline Ciprofloxacin & 17 & 1 & $98-102$ & 1 & 5.9 & 16 & 94.1 \\
\hline Doxycycline hyclate & 2 & & 95-102 & 0 & 0.0 & 2 & 100.0 \\
\hline Doxycycline & 85 & & $90-110$ & 48 & 56.5 & 37 & 43.5 \\
\hline Ofloxacin & 68 & & 99-101 & 2 & 2.9 & 66 & 97.1 \\
\hline Sulfamethoxazole & 124 & 1 & $90-110$ & 76 & 61.3 & 48 & 38.7 \\
\hline Tetracycline & 117 & & 96-102 & 51 & 43.6 & 66 & 56.4 \\
\hline Trimethoprim & 124 & 1 & $90-110$ & 60 & 48.4 & 64 & 51.6 \\
\hline Total & 1025 & 9 & & 408 & 39.8 & 617 & 60.2 \\
\hline
\end{tabular}

ascertain the authenticity of the sample; whether it was substandard or falsified cannot therefore be confidently ascertained.

The main problem identified was the presence of probably substandard, rather than falsified, antibiotics; $60.2 \%$ of the samples had units outside of the uniformity of the content limit range of the label claim. In the absence of chemical assays to distinguish degradation from poor factory production in field-collected samples, it is very difficult to distinguish failed samples as degraded or substandard, or both. ${ }^{32,60}$ There is an urgent need for research to develop such techniques. MS fingerprinting of degradation products may allow this distinction. ${ }^{61,62}$ An additional issue is that smaller companies may not have the human capacity, equipment and consumables to check the quality of the imported bulk API. For substandard medicines, the quality defect may have been in the API producer rather than the factory formulating the finished product. The storage conditions up to the time of purchase are unknown. Poor storage conditions may have contributed to degradation of APIs and excipients. Changes in crystalline morphology caused by high temperature can affect the dissolution or disintegration of the active ingredients, impairing bioavailability. ${ }^{63-66}$ This is also true 
Table 2. Units within, above and below the International Pharmacopoeia specifications of limit range of uniformity of content assay

\begin{tabular}{|c|c|c|c|c|c|c|c|c|c|}
\hline \multirow[b]{2}{*}{ API } & \multicolumn{2}{|c|}{ Good quality } & \multicolumn{2}{|c|}{ Under limit range } & \multicolumn{2}{|c|}{ Over limit range } & \multicolumn{2}{|c|}{ Under and over limit range } & \multirow[b]{2}{*}{ Total no. } \\
\hline & no. & $\%$ & no. & $\%$ & no. & $\%$ & no. & $\%$ & \\
\hline Amoxicillin & 30 & 13.3 & 36 & 16.0 & 151 & 67.1 & 8 & 3.6 & 225 \\
\hline Ampicillin & 137 & 53.5 & 24 & 9.4 & 88 & 34.4 & 7 & 2.7 & 256 \\
\hline Ceftriaxone & 3 & 42.9 & 4 & 57.1 & 0 & 0.0 & 0 & 0.0 & 7 \\
\hline Ciprofloxacin & 1 & 5.9 & 5 & 29.4 & 10 & 58.8 & 1 & 5.9 & 17 \\
\hline Doxycycline & 48 & 55.2 & 0 & 0.0 & 39 & 44.8 & 0 & 0.0 & 87 \\
\hline Ofloxacin & 2 & 2.9 & 18 & 26.5 & 39 & 57.4 & 9 & 13.2 & 68 \\
\hline Sulfamethoxazole & 76 & 61.3 & 43 & 34.7 & 5 & 4.0 & 0 & 0.0 & 124 \\
\hline Tetracycline & 51 & 43.6 & 11 & 9.4 & 55 & 47.0 & 0 & 0.0 & 117 \\
\hline Trimethoprim & 60 & 48.4 & 64 & 51.6 & 0 & 0.0 & 0 & 0.0 & 124 \\
\hline Total & 408 & 39.8 & 205 & 20.0 & 387 & 37.8 & 25 & 2.4 & 1025 \\
\hline
\end{tabular}

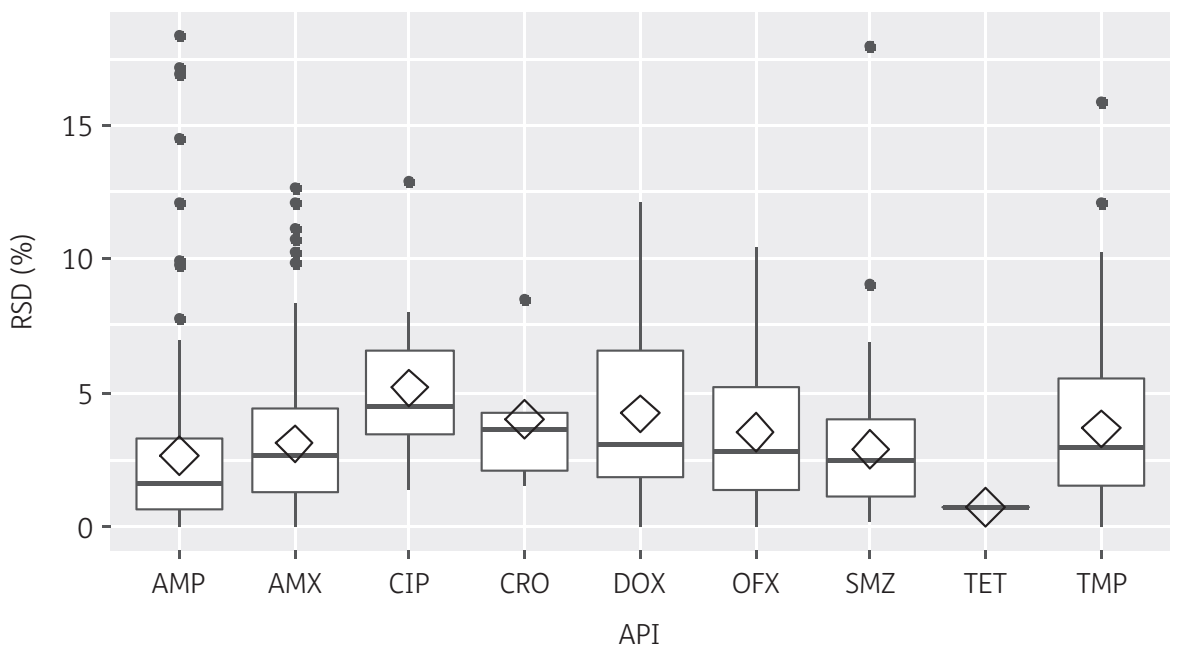

Figure 3. Interunit variability between units per sample measured as the RSD by medicine. It includes both intravenous and oral forms. Diamonds represent mean values and horizontal lines represent median values. AMP, ampicillin $(n=256)$; AMX, amoxicillin $(n=225)$; CRO, ceftriaxone $(n=7)$; CIP, ciprofloxacin ( $n=17)$; DOX, doxycycline $(n=87)$; OFX, ofloxacin $(n=68)$; SMZ, sulfamethoxazole $(n=124)$; TET, tetracycline $(n=117)$; TMP, trimethoprim $(n=124)$.
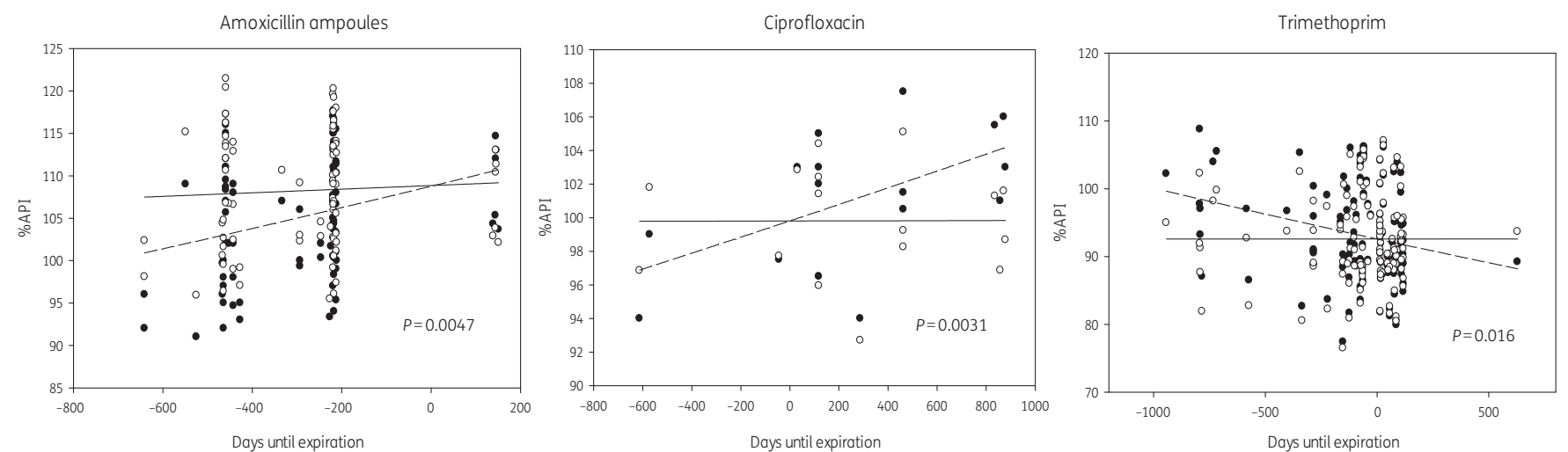

Figure 4. Stability plots showing changes in API content relative to expiration date. Dashed lines represent trends associated with these changes. The \%API values of medicines with significant slopes $(P<0.05)$ were normalized and are represented by solid lines. Amoxicillin ampoules, $n=246$; ciprofloxacin, $n=51$; trimethoprim, $n=372$. 
when analysing expired samples, as the medicine may or may not maintain its potency beyond the expiry date. Stability plots demonstrated potency before and after the expiry date and show the variability associated with the analysis as well as the tablets.

The significance of these data is important for individual patients as they risk impaired therapeutic efficacy and/or adverse drug reactions. Almost two-thirds of medicines outside the specification range had a high \%API concentration. This is of particularly great concern for medicines with narrow therapeutic indices, such as chloramphenicol, which can cause bone marrow toxicity and even death. Tetracycline is easily degraded under unfavourable storage conditions, resulting in potentially noxious degradation products. ${ }^{31,34}$ Even if clinical consequences are relatively rare, they would be very difficult to detect and manage in rural Laos. ${ }^{67}$

A significant minority of the samples (15.6\%) were sold loose with no labelling or manufacturing information. This finding is consistent with other surveys conducted in Laos. ${ }^{31,35}$ Inadequate labelling not only results in poor information on drug use, for patients and health workers, but it also provides opportunities for the sale of unregistered and falsified medicines. ${ }^{68}$ Eight samples had expired at the time of the survey when there should have been none.

Antimicrobials with low \%API, poor bioavailability or degradation may engender drug resistance. Modelling studies suggest that high $\beta$-lactam antibiotic doses at low frequencies produced more highly resistant Streptococcus pneumoniae strains, but at far lower prevalence than repeated exposure to subtherapeutic doses, which resulted in the highest prevalence of resistant strains. ${ }^{6,69}$

In addition, unregulated provision of antibiotics, dispensing of insufficient doses and the reduced adherence to complete dose regimens may contribute to the spread of antibiotic resistance. ${ }^{70}$ The high over-the-counter availability of antibiotics found in this survey suggests that overuse of antibiotics may be common. This problem becomes increasingly complex as many medicine sellers have not been trained in diagnosis and have limited knowledge on antibiotic posology and resistance.

Furthermore, anti-TB medicines, such as single agent isoniazid and rifampicin, were also sold by some outlets, even though fixed dose combination therapy for TB is available for free for patients through the Global Fund via the National TB Programme. The unregulated use of these monotherapies is likely to precipitate treatment failure and engender TB multidrug resistance.

Accurate prescribing decisions, appropriate treatment and rational use of drugs are major concerns among healthcare services in Laos. ${ }^{71}$ Nevertheless, enhanced pharmacy regulation, health education programmes and improvements in medicine labelling in Lao language are needed to promote appropriate antibiotic use.

\section{Limitations}

Itinerant drug sellers were not included in the survey, but they may stock anti-infective medicines and may reach remote communities. As the sale of medicines from unlicensed outlets is illegal, we have underestimated these sources. We did not examine the quality of antibiotics in the public sector.

Dissolution and disintegration tests were not performed, and the numbers of dosage units collected per sample were low. Medicines sold in small unlabelled plastic bags may already have expired at the time of sample collection and the storage conditions of the samples before collection are not known. That 31\% of samples were analysed after their expiry date is an important limitation and cautions against overinterpretation of these data. With the significant human investment needed to analyse samples, conducting analysis of many units before their expiry date is problematic; few papers describe the date of analysis in relation to sample expiry date.

The differences found in the packaging analysis suggest that samples may have been from a different manufacturer, brand or batch. Analysis of CD-3 data was impaired by difficulties in obtaining appropriate genuine samples for all the collected samples. Excipient variation between different manufacturers or the coating of the samples may have had an impact; or medicines may have been degraded due to poor storage conditions. Dose to dose variations within the samples were found, and it is unclear as to how many failed dosage units in a sample should be regarded as minimally acceptable. ${ }^{72}$

\section{Acknowledgements}

We greatly appreciate the support of the Lao Food and Drug Department, the Lao Bureau of Food and Drug Inspection, the Centre of Malariology, Parasitology and Entomology (CMPE), Lao PDR and their provincial and district health departments for their support and knowledge throughout the survey. We thank the Directors of Mahosot Hospital and the late Dr Rattanaphone Phetsouvanh and staff of the Microbiology Laboratory for their help. We also thank Anousone Duangnouvong, Danoy Chommanam and Outhevanh Kounnavongsa for their help with analysis and the scanning and logging of the medicines, Drs Serena Vickers and Céline Caillet for their help obtaining the pharmacopoeial limit ranges, and Dr Luis Merino-Martín for advice regarding statistical analysis.

\section{Funding}

The study was funded by the Project Priority Solidarity Funds (FSP) Mekong, with finance from the French Ministry of Foreign Affairs and International Development and the Wellcome Trust of Great Britain and the Artemisinin Combination Therapy Consortium (ACTC) via an award from the Bill and Melinda Gates Foundation to the London School of Hygiene and Tropical Medicine (LSHTM).

\section{Transparency declarations}

None to declare.

\section{Supplementary data}

Figures S1 to S3, Text S1, Form S1 and Tables S1 to S5 are available as Supplementary data at JAC Online.

\section{References}

1 Odermatt P, Ly S, Simmala C et al. Availability and costs of antiepileptic drugs and quality of phenobarbital in Vientiane municipality, Lao PDR. Neuroepidemiology 2007; 28: 169-74.

2 WHO. Antimicrobial Resistance Factsheet. http://www.who.int/media centre/factsheets/fs194/en/.

3 WHO. The Evolving Threat of Antimicrobial Resistance: Options for Action. http://apps.who.int/iris/bitstream/10665/44812/1/9789241503181_eng.pdf. 
4 WHO. WHO Global Strategy for Containment of Antimicrobial Resistance. http://www.who.int/drugresistance/WHO_Global_Strategy_English.pdf.

5 Sosa AJ. Antimicrobial Resistance in Developing Countries. New York: Springer, 2010. http://www.springer.com/gp/book/9780387893693.

6 Pisani E. Antimicrobial Resistance: What Does Medicine Quality Have to do With it? 2015. https://amr-review.org/sites/default/files/ElizabethPisani MedicinesQualitypaper.pdf.

7 Abdul-Aziz MH, Lipman J, Mouton JW et al. Applying pharmacokinetic/ pharmacodynamic principles in critically ill patients: optimizing efficacy and reducing resistance development. Semin Respir Crit Care Med 2015; 36: 136-53.

8 Leslie T, Kaur H, Mohammed $\mathrm{N}$ et al. Epidemic of Plasmodium falciparum malaria involving substandard antimalarial drugs, Pakistan, 2003. Emerg Infect Dis 2009; 15: 1753-9.

9 Nwokike J, Clark A, Nguyen PP. Medicines quality assurance to fight antimicrobial resistance. Bull World Health Organ 2018; 96: 135-7.

10 Delepierre A, Gayot A, Carpentier A. Update on counterfeit antibiotics worldwide; public health risks. Med Mal Infect 2012; 42: 247-55.

11 Johnston A, Holt DW. Substandard drugs: a potential crisis for public health. Br J Clin Pharmacol 2014; 78: 218-43.

12 Kelesidis T, Kelesidis I, Rafailidis PI et al. Counterfeit or substandard antimicrobial drugs: a review of the scientific evidence. J Antimicrob Chemother 2007; 60: 214-36.

13 Nickerson JW, Attaran A, Westerberg BD et al. Fatal bacterial meningitis possibly associated with substandard ceftriaxone-Uganda, 2013. MMWR Morb Mortal Wkly Rep 2016; 64: 1375-7.

14 Caudron JM, Ford N, Henkens M et al. Substandard medicines in resourcepoor settings: a problem that can no longer be ignored. Trop Med Int Health 2008; 13: 1062-72.

15 Yoshida N, Khan MH, Tabata H et al. A cross-sectional investigation of the quality of selected medicines in Cambodia in 2010. BMC Pharmacol Toxicol 2014; 15: 13.

16 Fadeyi I, Lalani M, Mailk N et al. Quality of the antibiotics-amoxicillin and co-trimoxazole from Ghana, Nigeria, and the United Kingdom. Am J Trop Med Hyg 2015; 92: 87-94.

17 Holmes AH, Moore LSP, Sundsfjord A et al. Understanding the mechanisms and drivers of antimicrobial resistance. Lancet 2016; 387: 176-87.

$18 \mathrm{Pan} \mathrm{H}$, Luo H, Chen S et al. Pharmacopoeial quality of antimicrobial drugs in southern China. Lancet Glob Health 2016; 4: e300-2.

19 Ndwigah S, Stergachis A, Abuga K et al. The quality of anti-malarial medicines in Embu County, Kenya. Malar J 2018; 17: 330.

20 Chikowe I, Bliese SL, Lucas S et al. Amoxicillin quality and selling practices in urban pharmacies and drug stores of Blantyre, Malawi. Am J Trop Med Hyg 2018; 99: 233-8.

21 Shakoor O, Taylor RB, Behrens RH. Assessment of the incidence of substandard drugs in developing countries. Trop Med Int Health 1997; 2: 839-45.

22 Newton PN, McGready R, Fernandez F et al. Manslaughter by fake artesunate in Asia-will Africa be next? PLoS Med 2006; 3: e197.

23 Newton PN, Green MD, Fernández FM et al. Counterfeit anti-infective drugs. Lancet Infect Dis 2006; 6: 602-13.

24 Newton PN, Fernandez FM, Plancon A et al. A collaborative epidemiological investigation into the criminal fake artesunate trade in South East Asia. PLoS Med 2008; 5: e32.

25 Lon CT, Tsuyuoka R, Phanouvong S et al. Counterfeit and substandard antimalarial drugs in Cambodia. Trans R Soc Trop Med Hyg 2006; 100: 1019-24.

26 WHO. Seventieth World Health Assembly Update, 29 May 2017. http:// www.who.int/mediacentre/news/releases/2017/dementia-immunization-ref uguees/en/.
27 Newton PN, Tabernero P, Dwivedi P et al. Falsified medicines in Africa: all talk, no action. Lancet Glob Health 2014; 2: e509-10.

28 Newton P, Proux S, Green M et al. Fake artesunate in southeast Asia. Lancet 2001; 357: 1948-50.

29 Dondorp AM, Newton PN, Mayxay M et al. Fake antimalarials in Southeast Asia are a major impediment to malaria control: multinational crosssectional survey on the prevalence of fake antimalarials. Trop Med Int Health 2004; 9: 1241-6.

30 Phanouvong $S$ et al. Why be concerned about the quality of antimalarial and ARV drugs? (Poster) In: Seventh International Congress on AIDS in Asia and the Pacific. Kobe, Japan, 2005.

31 Syhakhang L, Lundborg CS, Lindgren B et al. The quality of drugs in private pharmacies in Lao PDR: a repeat study in 1997 and 1999. Pharm World Sci 2004; 26: 333-8.

32 Keoluangkhot V, Green MD, Nyadong L et al. Impaired clinical response in a patient with uncomplicated falciparum malaria who received poor-quality and underdosed intramuscular artemether. Am J Trop Med Hyg 2008; 78: 552-5.

33 Aldhous P. Counterfeit pharmaceuticals: murder by medicine. Nature 2005; 434: 132-6.

34 Stenson B, Lindgren BH, Syhakhang L et al. The Quality of Drugs in Private Pharmacies in the Lao People's Democratic Republic. Int J Risk Safety Med 1998; 11: 243-9.

35 Yong YL, Plançon A, Lau YH et al. Collaborative health and enforcement operations on the quality of antimalarials and antibiotics in southeast Asia. Am J Trop Med Hyg 2015; 92: 105-12.

36 Sengaloundeth S, Green MD, Fernandez FM et al. A stratified random survey of the proportion of poor quality oral artesunate sold at medicine outlets in the Lao PDR-implications for therapeutic failure and drug resistance. Malar J 2009; 8: 172.

37 Tabernero P, Mayxay M, Culzoni MJ et al. A repeat random survey of the prevalence of falsified and substandard antimalarials in the Lao PDR: a change for the better. Am J Trop Med Hyg 2015; 92: 95-104.

38 Taylor RB, Shakoor O, Behrens RH et al. Pharmacopoeial quality of drugs supplied by Nigerian pharmacies. Lancet 2001; 357: 1933-6.

39 Quet F, Vlieghe E, Leyer C et al. Antibiotic prescription behaviours in Lao People's Democratic Republic: a knowledge, attitude and practice survey. Bull World Health Organ 2015; 93: 219-27.

40 Stoesser N, Crook DW, Moore CE et al. Characteristics of CTX-M ESBL-producing Escherichia coli isolates from the Lao People's Democratic Republic, 2004-09. J Antimicrob Chemother 2012; 67: 240-2.

41 Stoesser $N$, Xayaheuang $S$, Vongsouvath $M$ et al. Colonization with Enterobacteriaceae producing ESBLs in children attending pre-school childcare facilities in the Lao People's Democratic Republic. J Antimicrob Chemother 2015; 70: 1893-7.

42 WHO. World Malaria Report 2013. Country Profile Lao People's Democratic Republic. 2013. http://www.who.int/malaria/publications/country-profiles/pro file_lao_en.pdf.

43 WHO/MMP and Ministry of Public Health Lao PDR. National Strategy for Malaria Control and Pre-Elimination: Laos PDR 2011-2015. http://www.natio nalplanningcycles.org/sites/default/files/planning_cycle_repository/lao_peo ples_democratic_republic/laopdr_nationalstrategyformalariacontrola.pdf.

44 WHO. Lao People's Democratic Republic. http://www.who.int/countries/ lao/en/.

45 The World Bank. Rural Population (\% of Total Population), Lao PDR. http:// data.worldbank.org/indicator/SP.RUR.TOTL.ZS? locations=LA.

46 Stenson B, Tomson G, Syhakhang L. Pharmaceutical regulation in context: the case of Lao People's Democratic Republic. Health Policy Plan 1997; 12: $329-40$. 
47 Green MD, Nettey H, Rojas OV et al. Use of refractometry and colorimetry as field methods to rapidly assess antimalarial drug quality. J Pharm Biomed Anal 2007; 43: 105-10.

48 British Pharmacopoeia Commission. British Pharmacopoeia. Norwich, UK: The Stationery Office Limited, 1998.

49 European Pharmacopoeia. European Pharmacopoeia 6.0, Vol. 1. 2008: 1084. Strasbourg, France: Council of Europe.

50 Pharmacopeia US. Uniformity of Dosage Units. Chapter 905. http://www. pharmacopeia.cn/v29240/usp29nf24s0_c905.html.

51 United States Pharmacopeial Convention. The United States Pharmacopeia. USA: The National Formulary, 2003.

$52 \mathrm{WHO}$. The International Pharmacopoeia (Electronic Resource)= Pharmacopoeia Internationalis, 4th edn. 2006. Geneva: WHO.

53 The United States Pharmacopeial Convention. USP-NF General Chapter $<905>$ Uniformity of Dosage Units. https://www.uspnf.com/notices/retiredcompendial-notices/usp-nf-general-chapter-uniformity-dosage-units.

54 Ranieri N, Tabernero P, Green MD et al. Evaluation of a new handheld instrument for the detection of counterfeit artesunate by visual fluorescence comparison. Am J Trop Med Hyg 2014; 91: 920-4.

55 Newton PN, Lee SJ, Goodman C et al. Guidelines for field surveys of the quality of medicines: a proposal. PLoS Med 2009; 6: e52.

56 WHO. WHO Project for the Surveillance and Monitoring of SSFFC Medical Products. WHO Drug Information Quality and Safety of Medicines. 2013: 115. http://www.who.int/medicines/publications/druginformation/issues/DrugInfor mation2013_Vol27-2/en/index.html.

57 WHO. Rapid Alert System. Combating Counterfeit Medicines. WHO Western Pacific Region, 2014. http://www.counterfeitmedalert.info/.

58 Khan MH, Hatanaka K, Sovannarith T et al. Effects of packaging and storage conditions on the quality of amoxicillin-clavulanic acid-an analysis of Cambodian samples. BMC Pharmacol Toxicol 2013; 14: 33.

59 Laserson KF, Kenyon AS, Kenyon TA et al. Substandard tuberculosis drugs on the global market and their simple detection. Int J Tuberc Lung Dis 2001; 5: 448-54.

60 Van Assche K, Nebot Giralt A, Caudron JM et al. Pharmaceutical quality assurance of local private distributors: a secondary analysis in 13 low-income and middle-income countries. BMJ Glob Health 2018; 3: e000771.
61 Hall Z, Allan EL, van Schalkwyk DA et al. Degradation of artemisininbased combination therapies under tropical conditions. Am J Trop Med Hyg 2016; 94: 993-1001.

62 Hostetler DM, Dwivedi P, Green MD et al. High throughput quantitation of artesunate and its degradation products by flow injection gradient ratio standard addition mass spectrometry (FI-GRSA-MS). Anal Methods 2012; 4: 3392-8.

63 Singh S, Mohan B. A pilot stability study on four-drug fixed-dose combination anti-tuberculosis products. Int J Tuberc Lung Dis 2003; 7: 298-303.

64 Ballereau F, Prazuck T, Schrive I et al. Stability of essential drugs in the field: results of a study conducted over a two-year period in Burkina Faso. Am J Trop Med Hyg 1997; 57: 31-6.

65 Nazerali H, Hogerzeil HV. The quality and stability of essential drugs in rural Zimbabwe: controlled longitudinal study. BMJ 1998; 317: 512-3.

66 WHO. Accelerated Stability Studies of Widely Used Pharmaceutical Substances Under Simulated Tropical Conditions. 1986: 119. Geneva: WHO.

67 Caillet C, Sichanh C, Assemat G et al. Role of medicines of unknown identity in adverse drug reaction-related hospitalizations in developing countries: evidence from a cross-sectional study in a teaching hospital in the Lao People's Democratic Republic. Drug Saf 2017; 40: 809-21.

68 Keohavong B, Syhakhang L, Sengaloundeth S et al. Rational use of drugs: prescribing and dispensing practices at public health facilities in Lao PDR. Pharmacoepidemiol Drug Saf 2006; 15: 344-7.

69 Opatowski L, Mandel J, Varon E et al. Antibiotic dose impact on resistance selection in the community: a mathematical model of $\beta$-lactams and Streptococcus pneumoniae dynamics. Antimicrob Agents Chemother 2010; 54: 2330-7.

70 Khennavong M, Davone V, Vongsouvath M et al. Urine antibiotic activity in patients presenting to hospitals in Laos: implications for worsening antibiotic resistance. Am J Trop Med Hyg 2011; 85: 295-302.

71 WHO. Lao People's Democratic Republic Held its 1st Multi-Sectoral Workshop on Combatting Antimicrobial Resistance During the World Antibiotic Awareness Week. http://www.wpro.who.int/laos/mediacentre/releases/ 2016/20161216-waaw-2016/en/.

72 Venhuis BJ, Zwaagstra ME, Keizers PHJ et al. Dose-to-dose variations with single packages of counterfeit medicines and adulterated dietary supplements as a potential source of false negatives and inaccurate health risk assessments. J Pharm Biomed Anal 2014; 89: 158-65. 